\title{
Towards a Combinatorial Proof Theory
}

\author{
Benjamin Ralph ${ }^{1,2}$ and Lutz Straßburger ${ }^{1,2}$ \\ 1 Inria Saclay, 1 rue Honoré d'Estienne d'Orves, Palaiseau 91120, France \\ 2 LIX, Ecole Polytechnique, Route de Saclay, 91128 Palaiseau Cedex, France
}

\begin{abstract}
The main part of a classical combinatorial proof is a skew fibration, which precisely captures the behavior of weakening and contraction. Relaxing the presence of these two rules leads to certain substructural logics and substructural proof theory. In this paper we investigate what happens if we replace the skew fibration by other kinds of graph homomorphism. This leads us to new logics and proof systems that we call combinatorial.
\end{abstract}

Keywords: Proof Theory · Combinatorial Proofs · Deep Inference · Fibrations - Cographs

\section{Introduction}

Combinatorial proofs have been introduced by Hughes [17 to give a "syntaxfree" presentation for proofs in classical propositional logic. In doing so, they give a possible response to Hilbert's 24th problem of identity between proofs 29]: two proofs are the same if they have the same combinatorial proof [18|1|27].

In a nutshell, a classical combinatorial proof consists of two parts: (i) a linear part, and (ii) a skew fibration. The linear part encodes a proof in multiplicative linear logic (MLL), whose conclusion is given as a cograph, together with an equivalence relation on the vertices encoding the axiom links of the proof. A combinatorial correctness criterion for this linear part can be given by Retoré's critically corded condition [22. The skew fibration then maps this linear cograph to the cograph of the conclusion of the whole proof. This precisely captures the behaviour of weakening and contraction in a classical proof.

Recently, the theory of combinatorial proofs has been extended to intuitionistic propositional logic [16] and to relevant logics [2]. For intuitionistic logic, the linear part of a combinatorial proof has to be restricted to intuitionistic multiplicative linear logic (IMLL), and for relevant logic the skew fibration had to be restricted to a surjective weak fibration. This raises the question of what happens with other substructural logics that restrict (in their sequent calculus formulation) the use of contraction and weakening?

To answer this question, we need to address another issue first. Whereas the linear part of a combinatorial proof corresponds to well understood proof theory - there are sequent calculi and proof nets for most variants of MLL (intuitionistic/classical, commutative/non-commutative, with mix/without mix, etc.) - the skew fibration part is less obviously linked to established proof theory. In particular, the lack of any natural class of sequent calculus proofs equivalent 
to skew fibrations has lead to procrustean hacking away at either the sequent calculus side (via the Homomorphism Sequent Calculus in [18] or deep inference rules inside the sequent calculus in [1]), at the combinatorial proofs side (via lax combinatorial proofs in [18), or at both (via separated combinatorial/sequent calculus proofs in [7]).

To address this problem, we will in this paper use a pure deep inference system 1315$] 6[14$ to deal with weakening and contraction. This leads to different degrees of freedom for the weakening and contraction rules than in the sequent calculus. Whereas in the sequent calculus we can allow or forbid the rules on the left and/or on the right of the turnstile, in deep inference we have other choices. Besides allowing or forbidding rules, we can restrict the rules to atomic formulas or not, and to shallow contexts or not. Furthermore, deep inference systems with contraction and weaking also admit the medial rule, which implements the classical implication

$$
\mathrm{m} \frac{(A \wedge B) \vee(C \wedge D)}{(A \vee C) \wedge(B \vee D)}
$$

This rule is derivable if contraction and weakening are present in their general form [6. However, as soon as one of the restrictions mentioned above is applied, medial is no longer derivable. Thus, the presence or absence of medial is another degree of freedom in the design of a logical system. This creates a rich variety of structural proof systems, some of which are familiar to deep inference proof theorists, and some of which are novel.

Another reason for using deep inference is the availability of decomposition theorems $24|5| 23|28| 4 \mid 25$, which allow us to decompose a given formal derivation into several phases each using only a specific subset of the inference rules. Using techniques and results from work on linear rewriting systems [25|9,10]11] one can obtain decompositions that provide exactly the separation in the linear part and the contraction-weakening part of classical logic that is expressed by combinatorial proofs. This leads to a close correspondence between combinatorial proofs and deep inference (see also [26]).

On the other hand, we can study the effect on combinatorial proofs if we restrict the notion of skew fibration by set theoretic and graph theoretic means: we can demand injectivity, surjectivity, or bijectivity, or we can demand the skew fibration to be a proper fibration. Certain restrictions create already studied logics; some do not seem to correspond to logics studied in the literature. In fact, if the condition for the map can be checked in polynomial time (in its size) then we can speak of proof systems in the sense of Cook and Reckhow [8, and we will coin the term combinatorial proof systems.

As the main result of this paper we will establish a strong correspondence between these combinatorial proof systems and the structural proof systems that naturally arise from restricting contraction and weakening in a deep inference system, as described above.

We begin the paper in Section 2 by recalling the relation between formulas and cographs. Then, in Section 3, we recall standard classical combinatorial proofs, and in Section 4, we recall standard deep inference proof systems, using 
open deduction. Finally, in Section 5 we introduce all the necessary technical definitions that allow us to state our main result, Theorem 5.5. establishing the correspondence between homomophism classes on cographs and structural proof systems in open deduction. The remaining Sections 6, 7, and 8 are dedicated to the proof of that theorem.

\section{Formulas and Cographs}

In this paper, we restrict our attention to classical and substructural propositional logics: decidable logics freely generated from atoms and the two connectives $\{\wedge, \vee\}$.

Definition 2.1 We define an inexhaustible set of positive and negative atoms $\mathcal{A}=\{a, \bar{a}, b, \bar{b}, \ldots\}$, and a set of formulae, $\mathcal{F}$, generated from these atoms:

$$
A::=a|(A \wedge A)|(A \vee A)
$$

We omit parentheses when there is no ambiguity.

A logic $\mathcal{L}$ is defined by a valuation function $\mathcal{V}_{\mathcal{L}}: \mathcal{F} \rightarrow\{0,1\}$ selecting correct formulae. The valuation function for Classical Propositional Logic, $\mathcal{V}_{\mathrm{CPL}}$ is defined in the usual way.

Definition 2.2 A graph $G=\left\langle V_{G}, E_{G}\right\rangle$ consists of a set of vertices $V_{G}$ and a set of edges $E_{G}$ which are two-element subsets of $V_{G}$. We write $v w \in E_{G}$ for $\{v, w\} \in E_{G}$. Given a set $S$, a labelled, or $S$-labelled, graph adds a map $L_{G}: V_{G} \rightarrow$ $S$, mapping vertices to elements of $S$. We assume graphs are irreflexive: for all $v \in V_{G}, v v \notin E_{G}$. A graph $H$ is an induced subgraph of $G$ if $V_{H} \subseteq V_{G}$ and $E_{H}=\left\{v w \mid v, w \in V_{H}, v w \in E_{G}\right\}$.

There is a useful correspondence between $\mathcal{F}$ and a certain class of graphs, called cographs. First, we construct the map from formulae to cographs by defining graphical equivalents for the logical connectives.

Definition 2.3 Let $G, H$ be disjoint graphs. We define respectively their union and their join:

$$
\begin{aligned}
& G \vee H=\left\langle V_{G} \cup V_{H}, E_{G} \cup E_{H}\right\rangle \\
& G \wedge H=\left\langle V_{G} \cup V_{H}, E_{G} \cup E_{H} \cup\left\{v w \mid v \in V_{G}, w \in V_{H}\right\}\right\rangle
\end{aligned}
$$

Definition 2.4 We define the map $\mathfrak{G}$ from formulae to $\mathcal{A}$-labelled graphs as follows:

- For $a \in \mathcal{A}, \mathfrak{G}(a)=\langle v, \emptyset\rangle$, with $L(v)=a$.

$-\mathfrak{G}(A \vee B)=\mathfrak{G}(A) \vee \mathfrak{G}(B), \mathfrak{G}(A \wedge B)=\mathfrak{G}(A) \wedge \mathfrak{G}(B)$.

We call any $\mathcal{A}$-labelled graphs in the image of $\mathfrak{G}$ a cograph, and we denote the set of cographs as $\mathcal{G}$. For ease, we will write $V_{A}$ for $V_{\mathfrak{G}(A)}$ and $E_{A}$ for $E_{\mathfrak{G}(A)}$.

We can characterise cographs without constructing the formulae they map from. 
Definition 2.5 A graph $G$ of four distinct vertices $V_{G}=\{v, w, u, z\}$ with $v w, w u, u z \in E_{G}$ and $v u, v z, w z \notin E_{G}$ is called a $P_{4}$ graph.

$$
\begin{aligned}
& v-w \\
& u-z
\end{aligned}
$$

Proposition 2.6 Cographs are exactly those graphs that are $P_{4}$-free, i.e., they do not contain a $P_{4}$ graph as an induced subgraph.

Proof This has been proved in many places, e.g. [12.

Definition 2.7 A context $K\{\}$ is a function from $\mathcal{F} \rightarrow \mathcal{F}$, created by replacing exactly one instance of an atom in a formula with a hole \{\} .

Definition 2.8 We define the following two equivalence relations on formulae, with $\star \in\{\vee, \wedge\}$ :

$$
K\{A \star B\} \equiv_{C} K\{B \star A\} \quad K\{A \star(B \star C)\} \equiv_{A} K\{(A \star B) \star C\}
$$

The equivalence relation $\equiv$, formula equivalence, is the reflexive, symmetric and transitive closure of $\equiv_{C} \cup \equiv_{A}$.

This definition of formula equivalence coincides exactly with the equivalence classes induced by the cograph map $\mathfrak{G}$ :

Proposition 2.9 For all formulae, $A \equiv B$ iff $\mathfrak{G}(A)=\mathfrak{G}(B)$.

Proof Straightforward induction on the size of the formulas (see e.g. [12]).

\section{Combinatorial Proofs}

Usually, the linear (or multiplicative) part of a combinatorial proof is defined graph theoretically. Here, since we are concerned chiefly with the structural part, we simply use a sequent calculus.

Definition 3.1 We define the logic of MLL by the following one-sided sequent calculus system, with multiset sequents:

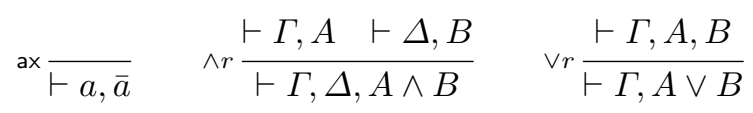

$\mathcal{V}_{\mathrm{MLL}}(A)=1$ iff there is a proof of $A$ in this sequent system.

Proposition 3.2 If $A \equiv B$ (and therefore $\mathfrak{G}(A)=\mathfrak{G}(B)$ ), then $\mathcal{V}_{\mathrm{MLL}}(A)=$ $\mathcal{V}_{\mathrm{MLL}}(B)$.

Proof Straightforward, since sequents are multisets.

Due to the above definition, we can define $\mathcal{V}_{\mathrm{MLL}}(G)=\mathcal{V}_{\mathrm{MLL}}(A)$ for any $A$ with $\mathfrak{G}(A)=G$. 

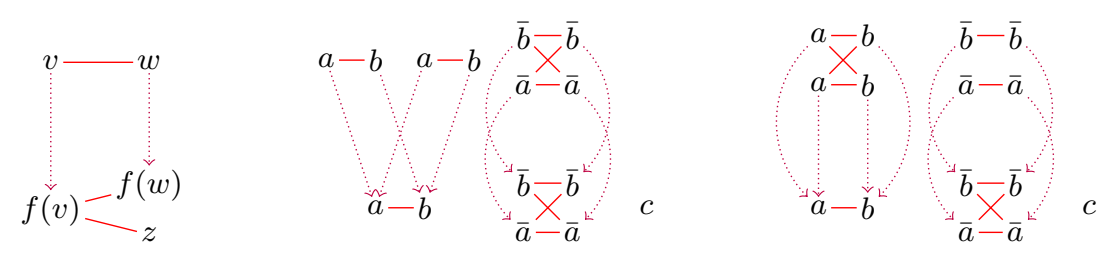

Fig. 1. On the left, we show a pictorial representation of the condition SF1. In the centre and on the right, two skew fibrations are shown, that are in fact combinatorial proofs of the formula $(a \wedge b) \vee((\bar{a} \vee \bar{b}) \wedge(\bar{a} \vee \bar{b})) \vee c$.

Definition 3.3 A graph homomorphism $f: V_{G} \rightarrow V_{H}$ is a map such that for all $e=v w \in E_{G}$, there is an edge $f(e)=f(v) f(w) \in E_{H}$. A skew fibration $f: G \rightarrow H$ is a graph homomorphism such that for every $v \in V_{G}, z \in V_{H}$ with $f(v) z \in E_{H}$, there is some $v w \in E_{G}$ such that:

(SF1) $f(w) z \notin E_{H}$.

Figure 1 illustrates this condition and gives two examples of skew fibrations.

With the technology that we have built up, we are able to define combinatorial proofs for classical logic.

Definition 3.4 Let $A \in \mathcal{F}$. $\phi=\left\langle G_{\phi}, f_{\phi}\right\rangle$ is a correct combinatorial proof [17] of $A$ for classical propositional logic if:

$-\mathcal{V}_{\mathrm{MLL}}\left(G_{\phi}\right)=1$

$-f_{\phi}: G_{\phi} \rightarrow \mathfrak{G}(A)$ is a skew fibration.

The two examples in Figure 1 are combinatorial proofs.

Remark 3.5 A graph theoretic counterpart to the first condition is that the cograph is non-empty critically chorded R\&B-cograph [22|26]. Nicely coloured graphs [17] correspond to theorems of MLL together with the mix rule.

\section{Open Deduction}

We introduce the deep inference proof formalism of open deduction [14].

Definition 4.1 The set of prederivations, $\mathcal{P}=\{\phi, \psi, \ldots\}$ is generated by the following grammar:

$$
\phi::=A|(\phi \wedge \phi)|(\phi \vee \phi) \mid \frac{\phi}{\phi}
$$

We define two functions premise and conclusion, $\mathrm{pr}, \mathrm{cn}: \mathcal{P} \rightarrow \mathcal{F}$,

$-\operatorname{pr}(A)=\operatorname{cn}(A)=A$ for $A \in \mathcal{F}$

$-\operatorname{pr}(\phi \star \psi)=\operatorname{pr}(\phi) \star \operatorname{pr}(\psi), \operatorname{cn}(\phi \star \psi)=\operatorname{cn}(\phi) \star \operatorname{cn}(\psi)$, for $\star \in\{\wedge, \vee\} ;$ 


$$
\operatorname{sw\downarrow } \frac{\operatorname{sc\downarrow } \frac{(a \wedge b) \vee(a \wedge b)}{a \wedge b} \vee((\bar{a} \vee \bar{b}) \wedge(\bar{a} \vee \bar{b}))}{(a \wedge b) \vee((\bar{a} \vee \bar{b}) \wedge(\bar{a} \vee \bar{b})) \vee c} \quad\left(\mathrm{ac \downarrow} \frac{a \vee a}{a} \wedge \mathrm{ac} \downarrow \frac{b \vee b}{b}\right) \vee \mathrm{sw \downarrow} \frac{(a \wedge \bar{a}) \vee(b \wedge \bar{b})}{\mathrm{m} \frac{(a \wedge \bar{a}) \vee(b \wedge \bar{b})}{(a \vee b) \wedge(\bar{a} \vee \bar{b})} \vee c}
$$

Fig. 2. Two simple derivations with the same conclusion $(a \wedge b) \vee((\bar{a} \vee \bar{b}) \wedge(\bar{a} \vee \bar{b}))$, the first with non-atomic shallow contraction and the second with medial and deep atomic contraction.

$$
-\operatorname{pr}\left(\frac{\phi}{\psi}\right)=\operatorname{pr}(\phi), \mathrm{cn}\left(\frac{\phi}{\psi}\right)=\mathrm{cn}(\psi)
$$

Definition 4.2 An inference rule $\rho$ is a polynomial-time decidable relation on $\mathcal{F}$. We write $\frac{A}{B} \rho$ if $\langle A, B\rangle \in \rho$. The equivalence rule is the formula equivalence relation as defined in Definition $\sqrt[2.8]{2}$, we write $\equiv \frac{A}{B}$ if $A \equiv B$. A proof system $\mathrm{S}$ is a finite set of inference rules, that (usually implicitly) contains the equivalence rule. The set of derivations, $\mathcal{D}_{\mathrm{S}} \subseteq \mathcal{P}$ of a proof system $\mathrm{S}$ is precisely the prederivations where vertical composition $\frac{\phi}{\psi}$ is restricted to cases where $\rho \frac{\operatorname{cn} \phi}{\operatorname{pr} \psi}$ is a correct instance for some $\rho \in \mathrm{S}$.

Remark 4.3 Since there is no need in this paper, we do not define proofs, i.e. derivations from no premise.

Definition 4.4 We define the structural rules of contraction, atomic contraction, weakening and medial:

$$
\mathrm{c \downarrow} \frac{A \vee A}{A} \quad \quad \mathrm{ac} \downarrow \frac{a \vee a}{a} \quad \mathrm{w} \downarrow \frac{B}{A \vee B} \quad \mathrm{~m} \frac{(A \wedge B) \vee(C \wedge D)}{(A \vee C) \wedge(B \vee D)}
$$

An instance of $c \downarrow, w \downarrow$, ac $\downarrow$ is called shallow if they are not in the scope of a conjunction. We denote such instances by sc $\downarrow$, sw $\downarrow$ and sac $\downarrow$ respectively. In Figure 2 we give two examples of derivations with these rules.

We are now almost ready to define the class of deep inference proofs that will correspond to combinatorial proofs. First, however, we define the notion of relative strength between proof systems.

Definition 4.5 Let $\mathrm{S}$ be a proof system and $\rho$ an inference rule. If for every instance $\rho \frac{A}{B}$ there is a derivation $\|_{B}^{A}$, we say $\rho$ is derivable for $\mathrm{S}$. If every rule $\rho \in \mathrm{S}^{\prime}$ is derivable for $\mathrm{S}$, then we write $\mathrm{S}^{\prime} \preceq \mathrm{S}$, where $\preceq$ is a partial order on proof systems. We write $\mathrm{S} \simeq \mathrm{S}^{\prime}$ if $\mathrm{S} \preceq \mathrm{S}^{\prime}$ and $\mathrm{S}^{\prime} \preceq \mathrm{S}$. 
Proposition $4.6 \quad \downarrow \downarrow$ is derivable for $\{\mathrm{ac} \downarrow, \mathrm{m}\}, \mathrm{m}$ is derivable for $\{\mathrm{w} \downarrow, \mathrm{c} \downarrow\}$. Hence $\{\mathrm{w} \downarrow, \mathrm{c} \downarrow\} \simeq\{\mathrm{w} \downarrow, \mathrm{m}, \mathrm{ac} \downarrow\}[\underline{6}]$.

Proof The first is proven by a simple structural induction on formulae, the key step being as follows:

$$
\mathrm{c} \downarrow \frac{(A \wedge B) \vee(A \wedge B)}{A \vee B} \longrightarrow \mathrm{m} \frac{(A \wedge B) \vee(A \wedge B)}{\mathrm{c} \downarrow \frac{A \vee A}{A} \wedge \mathrm{c} \downarrow \frac{B \vee B}{B}}
$$

The second needs only a simple rewrite:

$\mathrm{m} \frac{(A \wedge B) \vee(C \wedge D)}{(A \vee C) \wedge(B \vee D)} \rightarrow \mathrm{c \downarrow} \frac{\left(\mathrm{w} \downarrow \frac{A}{A \vee C} \wedge \mathrm{w} \downarrow \frac{B}{B \vee D}\right) \vee\left(\mathrm{w} \downarrow \frac{C}{A \vee C} \wedge \mathrm{w} \downarrow \frac{D}{B \vee D}\right)}{(A \vee C) \wedge(B \vee D)}$

A formal correspondence between derivations in open deduction and skew fibrations has been established in other works.

A

Proposition 4.7 Let $\phi \|\{w \downarrow, \mathrm{m}, \mathrm{ac} \downarrow\}$ be a derivation. Then there is a skew fibra$B$

tion $f: \mathfrak{G}(A) \rightarrow \mathfrak{G}(B)$. We denote this skew fibration $\mathfrak{G}(\phi)$.

Proposition 4.8 If $A, B \in \mathcal{F}$ and $f: \mathfrak{G}(A) \rightarrow \mathfrak{G}(B)$ is a skew fibration, then $A$

there is a derivation $\phi \|\left\{w_{\downarrow}, \mathrm{m}, \mathrm{ac} \downarrow\right\}$.

$B$

Proof Each proposition follows from one direction of [25, Theorem 7.8] and completeness and soundness of combinatorial proofs, respectively.

\section{Homomorphisms Classes and Structural Proof Systems}

In Section 2 we have shown the correspondence between formulae and cographs, and in Section 4 we have shown the correspondence between derivations in open deduction and skew fibrations. Now, we take this one step further, to a correspondence between deep inference proof systems and classes of homomorphisms, constructing two isomorphic lattices (partially ordered sets with meets and joins), of proof systems with respect to derivability, and homomorphism classes with respect to inclusion.

Definition 5.1 If $S \preceq\{w \downarrow, a c \downarrow, m\} \simeq\{w \downarrow, c \downarrow\}$, we say $S$ is structural. We denote the set of structural proof systems as $\mathcal{S}_{\downarrow}$. Let $S$ be a structural proof system. We define $\mathfrak{G}(\mathrm{S})=\left\{\mathfrak{G}(\phi) \mid \phi \in \mathcal{D}_{\mathrm{S}}\right\}$, the set of cograph homomorphisms generated from derivations in $\mathrm{S}^{3}$

\footnotetext{
3 The attentive reader might notice that $\mathfrak{G}$ can be seen as a functor between suitably defined categories. However, in order to make this paper accessible to a broader audience we decided not to use any category theoretical concepts here.
} 


\begin{tabular}{|l|l|l|l|}
\hline Iso & Isomorphisms & Bij & Bijections \\
\hline Inj & Injective Skew Fibrations & Sur & Surjective Skew Fibrations \\
\hline FInj & Full Injective Skew Fibrations & FSur & Full Surjective Skew Fibrations \\
\hline Fib & Fibrations & SFib & Surjective Fibrations \\
\hline FFib & Full Fibrations & FSkFib & Full Skew Fibrations \\
\hline FIFib & Full Injective Fibrations & FSFib & Full Surjective Fibrations \\
\hline WFib & Weak Fibrations & SWFib & Surjective Weak Fibrations \\
\hline FWFib & Full Weak Fibrations & FSWFib & Full Surjective Weak Fibrations \\
\hline
\end{tabular}

Fig. 3. Sets of Graph Homomorphisms

We now need to introduce some terminology to better characterise classes of homomorphisms.

Definition 5.2 A fibration $f: G \rightarrow H$ is a graph homomorphism such that for every $v \in V_{G}, z \in V_{H}$ with $f(v) z \in E_{H}$, there is some $v w \in E_{G}$ such that:

(F1) $f(w)=z$.

(F2) For all $v w^{\prime}$ with $f\left(w^{\prime}\right)=z$ we have $w^{\prime}=w$ (i.e. $w$ is unique).

A homomorphism is a weak fibration if it has has property (F1), but it may not have property (F2). Due to irreflexivity, a fibration is always a weak fibration which is always a skew fibration. A graph homomorphism $f: G \rightarrow H$ is full if $f(v) f(w) \in E_{H}$ implies $v w \in E_{G}$.

The first example in Figure 1 is a fibration, the second one is neither a fibration nor a weak fibration.

Definition 5.3 In Figure 3 we define sets of graph homomorphisms, all of which are subsets of the set SkFib of skew fibrations.

Proposition $5.4 \mathfrak{G}:\left\langle\mathcal{S}_{\downarrow}, \preceq\right\rangle \rightarrow\left\langle 2^{\mathrm{SkFib}}, \subseteq\right\rangle$ is an order-preserving injection from the set of structural proof systems into the set of subsets of cograph skew fibrations. In particular, $\mathfrak{G}(\{\mathrm{c} \downarrow, \mathrm{w} \downarrow\})=\mathfrak{G}(\{\mathrm{ac} \downarrow, \mathrm{m}, \mathrm{w} \downarrow\})=\mathrm{SkFib}$.

Proof We have that $\mathfrak{G}\left(\mathcal{S}_{\downarrow}\right) \subseteq$ SkFib from Proposition 4.7, and SkFib $\subseteq \mathfrak{G}\left(\mathcal{S}_{\downarrow}\right)$ from Proposition 4.8. Order preservation is clear from the definitions of $\preceq$ and $\mathfrak{G}$.

In the remainder of the paper we will be study the two lattices, $\left\langle\mathcal{S}_{\downarrow}, \preceq\right\rangle$ and $\left\langle 2^{\text {SkFib }}, \subseteq\right\rangle$; identifying which classes of homomorphisms correspond to structural proof systems and vice versa.

Theorem 5.5 The diagram in Figure 4 establishes corresponding points in the lattices $\left\langle\mathcal{S}_{\downarrow}, \preceq\right\rangle$ and $\left\langle 2^{\text {SkFib }}, \subseteq\right\rangle$, as explained in the caption. 


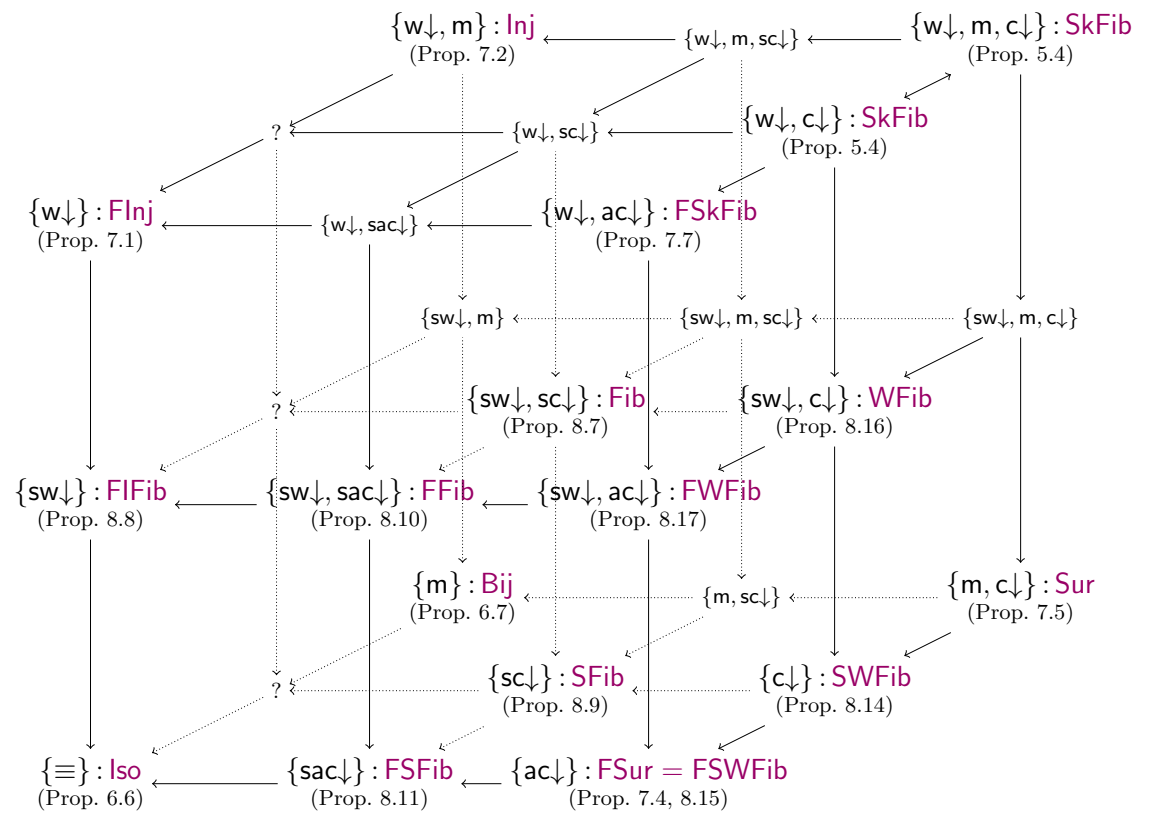

Fig. 4. At each point of the cube, the referenced proposition proves that $\mathfrak{G}$ maps from the proof system to the homomorphism class also at that point. Question marks refer to undefinable proof systems, and proof systems without propositions do not yet have proven homomorphism class equivalents - we do not suspect that any of these are of much interest.

\section{Basic Correspondences}

Just as we can compose derivations horizontally and vertically, we can also freely compose graph homomorphisms in corresponding ways.

Definition 6.1 Let $f: G \rightarrow H$ and $f^{\prime}: G^{\prime} \rightarrow H^{\prime}$ be graph homomorphisms. We define their union $f \vee f^{\prime}: G \vee G^{\prime} \rightarrow H \vee H^{\prime}$ and join $f \wedge f^{\prime}: G \wedge G^{\prime} \rightarrow H \wedge H^{\prime}$ such that the restrictions of each function to $G$ or $G^{\prime}$ are $f$ or $f^{\prime}$ respectively. We denote the identity functions as $\operatorname{id}_{G}: G \rightarrow G$ and empty functions as $\mathrm{e}_{G}: \emptyset \rightarrow G$.

In most cases, composing homomorphisms preserves their properties.

Proposition 6.2 Let $G, G^{\prime}, H, H^{\prime}$ be cographs.

1. Any isomorphism $i: G \rightarrow H$ is a fibration and thus also a skew fibration.

2. The map $\mathrm{w} \downarrow=i d_{G} \vee e_{H}: G \rightarrow G \vee H$, is a full injective fibration.

3. The map $\mathrm{\downarrow} \downarrow: G \vee G \rightarrow G$, which acts as the identity on each copy of $G$, is a full surjective fibration.

4. The map $\mathrm{m}:(G \wedge H) \vee\left(G^{\prime} \wedge H^{\prime}\right) \rightarrow\left(G \vee G^{\prime}\right) \wedge\left(H \vee H^{\prime}\right)$ which acts as the identity on $G, G^{\prime}, H, H^{\prime}$, is a bijective skew fibration (but not a fibration). 
5. If $f: G \rightarrow H$ and $f^{\prime}: G^{\prime} \rightarrow H^{\prime}$ are skew fibrations (respectively fibrations, injections, surjections, bijections or full) then $f \vee f^{\prime}: G \vee G^{\prime} \rightarrow H \vee H^{\prime}$ is a skew fibration (respectively fibration, injection, surjection, bijection or full).

6. If $f: G \rightarrow H$ and $f^{\prime}: G^{\prime} \rightarrow H^{\prime}$ are skew fibrations (respectively injections, surjections, bijections or full) then $f \wedge f^{\prime}: G \wedge G^{\prime} \rightarrow H \wedge H^{\prime}$ are skew fibration (respectively injection, surjection, bijection or full). This property does not hold for fibrations.

7. If $f: G \rightarrow G^{\prime}$ and $g: G^{\prime} \rightarrow H$ are skew fibrations (respectively fibrations, injections, surjections, bijections or full), then $g \circ f: G \rightarrow H$ is a skew fibration (respectively fibration, injection, surjection, bijection or full).

Proof Omitted but straightforward.

Before establishing the correspondences, we will first introduce some useful results from work investigating medial as a rewriting rule.

$A$

Theorem 6.3 [25, Theorem 5.1] There is a derivation $\|\{\mathrm{m}\}$ iff the following $B$

properties hold of $\mathfrak{G}(A)=\left\langle V_{A}, E_{A}\right\rangle$ and $\mathfrak{G}(B)=\left\langle V_{B}, E_{B}\right\rangle$.

1. $V_{A}=V_{B}$ and $E_{A} \subseteq E_{B}$

2. For all $a, d \in V_{A}$, s.t. $a d \in E_{B} \backslash E_{A}$, there are $b, c \in V_{A}$ s.t.

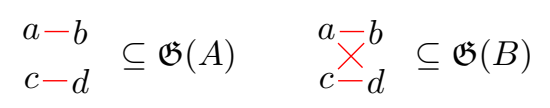

Using this theorem, it can be shown that skew fibrations correspond to a further stratified subclass of decomposed derivation.

Definition 6.4 A derivation in the following form is said to be structurally decomposed.

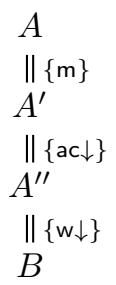

Theorem 6.5 There is a skew fibration $\mathfrak{G}(A) \rightarrow \mathfrak{G}(B)$ iff there is a structurally A

decomposed derivation $\|\{\mathrm{m}, \mathrm{ac} \downarrow, \mathrm{w} \downarrow\}$.

$B$

Proof [25, Theorem 7.8] A refinement of Propositions 4.7 and 4.8 .

Using this theorem, we can prove our first correspondences.

Proposition 6.6 $\mathfrak{G}(\{\equiv\})=$ Iso. 
Proof Corollary of Proposition 2.9.

The next proposition, that the proof system $\{\mathrm{m}\}$ corresponds to bijective skew fibrations, has been informally noted by Hughes [18, Section 9].

Proposition 6.7 $\mathfrak{G}(\{\mathrm{m}\})=\mathrm{Bij}$

Proof We get inclusion from Proposition 6.2 parts 4,5,6 and 7. For equality, we observe that if there is a bijective skew fibration from $\mathfrak{G}(A)$ to $\mathfrak{G}(B)$, then by

Theorem 6.5 we must have a structurally decomposed derivation $\left.\begin{array}{c}A \\ \phi\end{array}\right]\{\mathrm{m}, \mathrm{ac} \downarrow, \mathrm{w} \downarrow\}$.

Since any instance of ac $\downarrow$ in $\phi$ would break injectivity, and any instance of $w \downarrow$

$A$

would break surjectivity, we must have that $\phi \|\{m\}$.

\section{$7 \quad$ Restricting $\mathbf{c} \downarrow$ or $\mathbf{w} \downarrow$ : Affine and Relevance Logic}

Certain correspondences between proof systems and homomorphism classes have already been established in [25, [18] and [2] and others are simple corollaries of these.

Proposition 7.1 [25, Proposition 7.6] $\mathfrak{G}(\{w \downarrow\})=$ FInj.

Proposition $7.2 \mathfrak{G}(\{w \downarrow, m\})=\operatorname{lnj}$.

Proof Again, we get inclusion from Proposition 6.2 For equality, we observe that if there is an (full) injective skew fibration from $\mathfrak{G}(A)$ to $\mathfrak{G}(B)$, then by Theorem 6.5 we must have a structurally decomposed derivation $\phi \|$. Since any $B$

instance any instance of ac $\downarrow$ would break injectivity, we must have that

$$
\begin{gathered}
A \\
\phi=\phi_{1} \|\{\mathrm{m}\} \\
A^{\prime} \\
\phi_{2} \|\{\mathrm{w} \downarrow\} \\
B
\end{gathered}
$$

This gives us that $\mathfrak{G}(\{\mathrm{w} \downarrow, \mathrm{m}\})=\operatorname{Inj}$. For $\mathfrak{G}(\{\mathrm{w} \downarrow\})=$ Flnj, we observe that if $\phi$ contains an instance of medial, then, by Theorem 6.3 there is some $\left(\mathfrak{G}\left(\phi_{1}\right)(v)\right)\left(\mathfrak{G}\left(\phi_{1}\right)(w)\right) \in E_{A^{\prime}}$ such that $v w \notin E_{A}$. Since weakenings do not alter edges between existing vertices in the cographs, $v w \in E_{B}$, and therefore $\mathfrak{G}(\phi)$ is not full.

Remark 7.3 Allowing weakening but not contraction gives us affine logic. Therefore, insisting that the skew fibrations of combinatorial proofs are fully injective leads to combinatorial proofs for affine logic [18. 
Proposition 7.4 [25, Proposition 7.6] $\mathfrak{G}(\{\mathrm{ac} \downarrow\})=$ FSur $=$ FSWFib.

Proposition $7.5 \mathfrak{G}(\{\mathrm{ac} \downarrow, \mathrm{m}\})=$ Sur.

Proof Once more, we get inclusion from Proposition 6.2 For equality, we observe that if there is a bijective skew fibration from $\mathfrak{G}(A)$ to $\mathfrak{G}(B)$, then by Theorem

6.5 , we must have a structurally decomposed derivation ${ }_{B}^{A}$. Since any instance of $\mathbf{w} \downarrow$ would break surjectivity, we must have if $\mathfrak{G}(\phi)$ is a surjection, then

$$
\begin{gathered}
A \\
\phi=\begin{array}{c}
\phi_{1} \|\{\mathrm{m}\} \\
A^{\prime} \\
\phi_{2} \|\{\mathrm{ac} \downarrow\} \\
B
\end{array}
\end{gathered}
$$

This gives us $\mathfrak{G}(\{\mathrm{ac} \downarrow, \mathrm{m}\})=$ Sur. For $\mathfrak{G}(\{\mathrm{w} \downarrow\})=$ FSur, we observe that if $\phi$ contains an instance of medial, then, by Theorem 6.3 there is some $\left(\mathfrak{G}\left(\phi_{1}\right)(v)\right)\left(\mathfrak{G}\left(\phi_{1}\right)(w)\right) \in E_{A^{\prime}}$ such that $v w \notin E_{A}$. Since atomic contractions only contract vertices with no edge between them, the images of $v$ and $w$ under $\mathfrak{G}(\phi)$ are distinct and $(\mathfrak{G}(\phi)(v))(\mathfrak{G}(\phi)(w)) \in E_{B}$, and so $\mathfrak{G}(\phi)$ is not full.

Remark 7.6 Adding contraction but not weakening to MLL gives us relevance logic. Therefore, insisting that the skew fibrations of combinatorial proofs are surjective leads to combinatorial proofs for relevance logic 218 .

Just leaving out medial, we get full skew fibrations.

Proposition $7.7 \quad \mathfrak{G}(\{a c \downarrow, w \downarrow\})=$ FSkFib.

Proof Inclusion follows from Proposition 6.2 For equality, let $f: \mathfrak{G}(A) \rightarrow \mathfrak{G}(B)$ be a full skew fibration, and consider its corresponding structurally decomposed derivation:

$$
\begin{gathered}
A \\
\phi=\begin{array}{c}
\phi_{1} \|\{\mathrm{m}\} \\
A^{\prime} \\
\phi_{2} \|\{\mathrm{ac} \downarrow, \mathrm{w} \downarrow\} \\
B
\end{array}
\end{gathered}
$$

Assume there is some $\mathfrak{G}(\phi)(v w) \in E_{B}$ with $v w \notin E_{A^{\prime}}$. From the inclusion result, we have that $\mathfrak{G}\left(\phi_{2}\right)$ is a full skew fibration. In particular, since $\mathfrak{G}(\phi)(v w) \in E_{B}$, we have $\mathfrak{G}\left(\phi_{1}\right)(v w) \in E_{A}^{\prime}$. Therefore $\phi_{1}$ must contain at least one medial rule.

\section{Restricting to Shallow Inference: A Logic of Fibrations}

We now come on to logics not yet studied: what happens if we do not insist on either injectivity or surjectivity, but that the skew fibration is a graph fibration? 


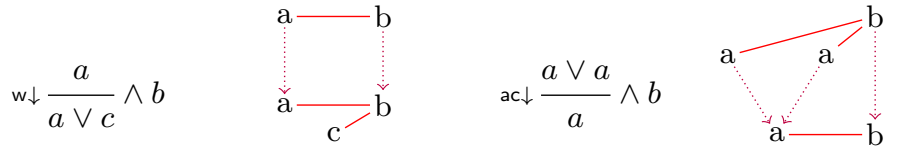

Fig. 5. Simple examples of skewed fibrations

It is instructive to turn to the simplest possible examples that are skew fibrations but not fibrations, in Figure 5. The left hand derivation fails condition $\mathbf{F 1}$ and the right hand derivation fails F2. In both cases, it is precisely the deepness of the rules that breaks each condition: if the contraction or weakening was in a disjunction with $b$, both would still be fibrations.

Definition 8.1 A path $v_{0} \ldots v_{n}$ in a graph $G$ is a sequence of vertices such that $v_{i} v_{i+1} \in E_{G}$ for $0 \leq i<n$. Two vertices of a graph $v, w \in V_{G}$ are connected if there is a path from $v$ to $w$. A graph (or subgraph) $G$ is connected if any two vertices in $G$ are connected. A subset $V^{\prime} \subseteq V_{G}$ is connected if there is a path between any two vertices in $V^{\prime}$ (the path does not need to stay in $V^{\prime}$ ). A maximal connected subset of vertices is called a component.

Proposition 8.2 Let $G$ be a cograph. If $v$ and $w$ are connected, then either $v w \in E_{G}$, or there is some $z \in V_{G}$ with $v z, z w \in E_{G}$.

Proof If the shortest path between $v$ and $w$ has three edges or more, then the first four vertices in that path will form a $P_{4}$ subgraph.

Proposition 8.3 Let $f: G \rightarrow H$ be a fibration between cographs. If $v, w \in G$ and $v \neq w$ and $f(v)=f(w)$, then $v$ and $w$ are in different components.

Proof Assume $v$ and $w$ are connected, so, since $f$ is a homomorphism, and $f(v) f(w) \notin E_{G}$ due to irreflexivity, there must be some $z \in G$ with $v z, z w \in G$, breaking the uniqueness property (F2) at $z$.

Proposition 8.4 Let $f: G \rightarrow H$ be a fibration between cographs. If $v, w \in H$ are connected, then either they are both in the image of $f$ or both not.

Proof We assume (WLOG) that $v w \in H, v$ is in the image of $f$, but $w$ is not, the fibration property (F1) breaks at $v$.

Proposition 8.5 If $f: G \rightarrow H$ is a fibration, and $H_{1}$ is a component of $H$, then $f^{-1}\left(H_{1}\right)$ is the union of zero or more copies of $H_{1}$.

Proof If some vertex in $H_{1}$ has a non-empty pre-image in $f$ then, by Proposition 8.4, every vertex does. Let $v^{\prime} \in H_{1}$. By Proposition 8.3, each vertex $v \in f^{-1}\left(v^{\prime}\right)$ is in a different component, and for each edge $v^{\prime} w_{i}^{\prime}$ and vertex $v$ there is a unique pre-image $v w_{i}$. Thus we can progressively recreate the whole component from a single vertex. 
Example 8.6 In Figure 2, the $c \downarrow$ in the left hand proof is shallow, but the two instances of ac $\downarrow$ in the right hand proof are not.

Proposition $8.7 \mathfrak{G}(\{s c \downarrow, s w \downarrow\})=$ Fib.

Proof Inclusion from Proposition 6.2, as usual, noting that we forbid horizontal composition by $\wedge$. For equality, take a fibration $f: \mathfrak{G}(A) \rightarrow \mathfrak{G}(B)$. Write $B$ as $B_{1} \vee \ldots \vee B_{n}$, where each $B_{i}$ is such that $\mathfrak{G}\left(B_{i}\right)$ is a component of $B$. Following Proposition 8.5 let $m_{i} \geq 0$ be the number of pre-images $\mathfrak{G}\left(B_{i}\right)$ has in $B$. We can rearrange the $B_{i}$ such that there is some $k$ with $n_{i}=0$ iff $i \leq k$. Then, we can construct the following derivation:

$$
\phi=\mathrm{sw \downarrow}_{\downarrow^{k-1}} \frac{\mathrm{sc}^{n} \frac{B_{k+1} \vee \ldots \vee B_{k+1}}{B_{k+1}} \vee \ldots \vee \mathrm{sc} \downarrow^{n} \frac{B_{n} \vee \ldots \vee B_{n}}{B_{n}}}{B_{1} \vee \ldots \vee B_{k} \vee \ldots \vee B_{k+1} \vee \ldots \vee B_{n}}
$$

where $\mathfrak{G}(\phi)=f$.

Proposition $8.8 \mathfrak{G}(\{s w \downarrow\})=$ FIFib.

Proposition $8.9 \quad \mathfrak{G}(\{\mathrm{sc} \downarrow\})=$ SFib.

Proposition $8.10 \mathfrak{G}(\{\mathrm{sw} \downarrow, \mathrm{sac} \downarrow\})=$ FFib.

Proposition 8.11 $\mathfrak{G}(\{\operatorname{sac} \downarrow\})=$ FSFib.

Proof All four are straightforward corollaries of Proposition 8.7.

We can adapt the above approach slightly for the case with weak fibrations.

Definition 8.12 Let $f: \mathfrak{G}(A) \rightarrow \mathfrak{G}(B)$ be a weak fibration, if $C$ is a subformula of $B$ with $\mathfrak{G}(C)$ a connected subgraph but $f^{-1}(\mathfrak{G}(C))$ not a connected subgraph, we say that $C$ is a contracted subformula. If there is no larger subfomula of $B$ with this property, we say that $C$ is a maximal contracted subformula.

Proposition 8.13 Let $f: \mathfrak{G}(A) \rightarrow \mathfrak{G}(B)$ be a surjective weak fibration, with $B=K_{B}\{C\}$ and $C$ a maximal contracted subformula of $B$. Define $B^{\prime}=$ $K_{B}\{C \vee C\}$. Then we can find a surjective weak fibration $f^{\prime}: \mathfrak{G}(A) \rightarrow \mathfrak{G}\left(B^{\prime}\right)$.

Proof If $\mathfrak{G}(C)$ is a component of $\mathfrak{G}(B)$, then it is straightforward. If not, then since $C$ is a contracted subformula, $f^{-1}(\mathfrak{G}(C))$ is a disconnected subgraph of $\mathfrak{G}(A)$. Denote the components of $f^{-1}(\mathfrak{G}(C))$ as $C_{1}, \ldots, C_{n}$, and define $C_{l}=\left\{v_{l} \mid\right.$ $v \in C\}$ and $C_{r}=\left\{v_{r} \mid v \in C\right\}$. We have:

$$
V_{A}=V_{K_{A}} \cup \bigcup_{1}^{n}\left(V_{C_{i}}\right) \quad V_{B}=V_{K_{B}} \cup V_{C} \quad V_{B}^{\prime}=V_{K_{B}} \cup V_{C_{l}} \cup V_{C_{r}}
$$

We define the homomorphisms $f^{\prime}: \mathfrak{G}(A) \rightarrow \mathfrak{G}(B)$ and $c: \mathfrak{G}\left(B^{\prime}\right) \rightarrow \mathfrak{G}(B)$ :

$$
f^{\prime}(v)=\left\{\begin{array}{l}
f(v): v \in V_{K_{A}} \\
v_{l} \quad: v \in V_{C_{1}} \\
v_{r} \quad: v \in V_{C_{i}}, i>1
\end{array}\right\} \quad c(v)=\left\{\begin{array}{l}
v: v \in V_{K_{B}} \\
w: v \in V_{C_{l}}, v=w_{l} \\
w: v \in V_{C_{r}}, v=w_{r}
\end{array}\right\}
$$



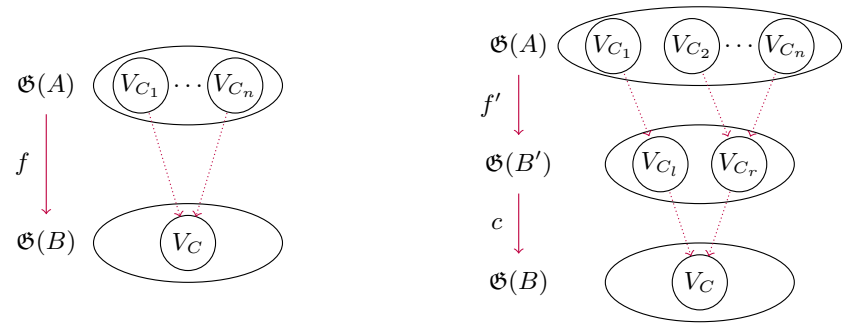

Fig. 6. Constructing $f^{\prime}$ in Proposition 8.13

Since $f$ is a homomorphism and a surjective weak fibration, $f\left(C_{i}\right)=C$ for every $1 \leq i \leq n$, so $f=c f^{\prime}$ and $f^{\prime}$ is surjective. We now need to show that $f^{\prime}$ is a weak fibration. Let $f^{\prime}(w) z \in E_{B^{\prime}}$, we need to show that there is some $\hat{z}$ with $w \hat{z} \in E_{A}$ and $f^{\prime}(\hat{z})=z$. The case where both $f^{\prime}(w), z \in V_{K_{B}}$ is trivial, as are the cases where $f^{\prime}(w), z \in V_{C_{l}}$ or $f^{\prime}(w), z \in V_{C_{r}}$.

If $f^{\prime}(w) \in V_{C_{l}}$ (WLOG) and $z \in V_{K_{B^{\prime}}}$, then we have $f(w) z \in E_{B}$, and since $f$ is a weak fibration, we have $\hat{z}$ with $f(\hat{z})=z$ and $w \hat{z} \in E_{A}$. As $z \in V_{K_{B}}$, we also have that $f^{\prime}(\hat{z})=z$.

Finally, if $f^{\prime}(w) \in V_{K_{B^{\prime}}}$ and $z \in V_{C_{l}}$ (WLOG), then we have $f(w) c(z) \in E_{B}$. As $f$ is a weak fibration, then we have some $\hat{z}_{i} \in C_{i}$ with $f\left(\hat{z}_{i}\right)=z, z_{i} \in C_{i}$ and $\hat{z}_{i} w \in E_{A}$. If $i=1$ we are done. If not, we need to show that $\hat{z}_{1} w \in$ $E_{A}$. Since $C$ is maximal, we must have that $f^{-1}\left(V_{C} \cup\{f(w)\}=\bigcup_{1}^{n}\left(V_{C_{i}}\right) \cup\right.$ $f^{-1}(f(w))$ is connected. Therefore, by Proposition 8.2, since $\hat{z}_{1} \hat{z}_{i} \notin E_{A}$ we must have $\hat{z}_{1} w^{\prime}, w^{\prime} \hat{z}_{i}$, for some $w^{\prime}$ where $f\left(w^{\prime}\right)=f(w)$. But then, for $\hat{z}_{1}, \hat{z}_{i}, w, w^{\prime}$ not to be a $P_{4}$ subgraph, we need $\hat{z}_{1} \hat{z}_{i}, w w^{\prime}$ or $\hat{z}_{1} w$ to be in $V_{A}$. Since $f(w)=f\left(w^{\prime}\right)$ and $f\left(\hat{z}_{1}\right)=f\left(\hat{z}_{i}\right)$, we must have $\hat{z}_{1} w \in E_{A}$. Therefore $f^{\prime}$ is a weak fibration.

Proposition $8.14 \mathfrak{G}(\{\mathfrak{c} \downarrow\})=$ SWFib.

Proof Inclusion from Proposition 6.2 noting that horizontal composition of derivations only violates condition $(\mathbf{F} 1)$ if the derivation contains weakenings. For equality, consider a surjectve weak fibration $f: \mathfrak{G}(A) \rightarrow \mathfrak{G}(B)$. We build the A

derivation $\|\{c \downarrow\}$ working up by contracting on maximal contracted subformulae $B$

of $B$ using Proposition 8.13

We can now prove a simple but purely graph theoretic result using the correspondence with structural proof systems.

Proposition 8.15 Every full surjective skew fibration is a weak fibration, i.e. FSur $=$ FSWFib.

Proof By definition FSWFib $\subseteq$ FSur. Since $\mathfrak{G}(\mathrm{ac} \downarrow)=$ FSur, $\{\mathrm{ac} \downarrow\} \preceq\{c \downarrow\}$ and $\mathfrak{G}(c \downarrow)=$ SWFib, we must have that FSur $\subseteq$ SWFib.

Proposition 8.16 $\mathfrak{G}(\{s w \downarrow, c \downarrow\})=$ WFib. 
Proposition $8.17 \mathfrak{G}(\{s w \downarrow, a c \downarrow\})=$ FWFib.

Proof Simple corollaries of Propositions 8.14, 8.7 and 7.4

\section{Conclusion}

Cographs can describe formulas without using a syntax tree. Even though this concept has been known for more than 50 years, these formulas without syntax have been used for proof theoretical considerations first by Retoré [20|21|22] and Guglielmi [13. Hughes [17] provided the next natural step by studying combinatorial proofs as proofs without syntax, as they describe proofs without the syntax of a proof tree. In this paper we have generalized this further to proof systems without syntax, using graph homomorphism classes instead of inference rules.

Summarizing the main theorem leads to the following slogans relating homomorphism classes and proof systems:

$$
\begin{aligned}
\text { No Weakening } & =\text { Surjectivity } \\
\text { No Contraction } & =\text { Injectivity } \\
\text { Atomic Contraction } & =\text { Fullness } \\
\text { Shallow Inference } & =\text { Fibrations } \\
\text { Deep Inference } & =\text { Skew Fibrations }
\end{aligned}
$$

An important line of future research is the extension of these results to modal logics [3] and first-order logic [19].

\section{Acknowledgements}

This research was supported by the ANR-FWF International Project ANR-15CE25-0014-01 FISP. We would also like to thank the anonymous referees for their helpful comments.

\section{References}

1. Matteo Acclavio and Lutz Straßburger. From syntactic proofs to combinatorial proofs. In International Joint Conference on Automated Reasoning, pages 481497. Springer, 2018.

2. Matteo Acclavio and Lutz Straßburger. On Combinatorial Proofs for Logics of Relevance and Entailment. In Rosalie Iemhoff, Michael Moortgat, and Ruy de Queiroz, editors, Logic, Language, Information, and Computation, pages 1-16. Springer Berlin Heidelberg, 2019.

3. Matteo Acclavio and Lutz Straßburger. On Combinatorial Proofs for Modal Logic. In International Conference on Automated Reasoning with Analytic Tableaux and Related Methods, London, 2019. Springer. 
4. Andrea Aler Tubella, Alessio Guglielmi, and Benjamin Ralph. Removing Cycles from Proofs. In Valentin Goranko and Mads Dam, editors, 26th EACSL Annual Conference on Computer Science Logic (CSL 2017), volume 82 of Leibniz International Proceedings in Informatics (LIPIcs), pages 9:1-9:17. Schloss DagstuhlLeibniz-Zentrum fuer Informatik, 2017.

5. Kai Brünnler. Locality for classical logic. Notre Dame Journal of Formal Logic, 47(4):557-580, 2006.

6. Kai Brünnler and Alwen Tiu. A local system for classical logic. In Logic for Programming, Artificial Intelligence, and Reasoning, volume 2250, pages 347-361. Springer, 2001.

7. Alessandra Carbone. A new mapping between combinatorial proofs and sequent calculus proofs read out from logical flow graphs. Information and Computation, 208(5):500-509, May 2010.

8. Stephen A. Cook and Robert A. Reckhow. The relative efficiency of propositional proof systems. The journal of symbolic logic, 44(01):36-50, 1979.

9. Anupam Das. Rewriting with Linear Inferences in Propositional Logic. In RTA, pages 158-173, 2013.

10. Anupam Das and Lutz Straßburger. No complete linear term rewriting system for propositional logic. In 26th International Conference on Rewriting Techniques and Applications (RTA), Leibniz International Proceedings in Informatics (LIPIcs). Schloss Dagstuhl-Leibniz-Zentrum für Informatik, 2015.

11. Anupam Das and Lutz Straßburger. On linear rewriting systems for Boolean logic and some applications to proof theory. Logical Methods in Computer Science, 12, 2016.

12. Richard J. Duffin. Topology of series-parallel networks. Journal of Mathematical Analysis and Applications, 10(2):303-318, 1965.

13. Alessio Guglielmi. A system of interaction and structure. ACM Transactions on Computational Logic, 8(1), January 2007.

14. Alessio Guglielmi, Tom Gundersen, and Michel Parigot. A Proof Calculus Which Reduces Syntactic Bureaucracy. Proceedings of the 21st International Conference on Rewriting Techniques and Applications (RTA'10), 6:135-150, 2010.

15. Alessio Guglielmi and Lutz Straßburger. Non-commutativity and MELL in the calculus of structures. In International Workshop on Computer Science Logic, pages 54-68. Springer, 2001.

16. Willem Heijltjes, Dominic J. D. Hughes, and Lutz Straßburger. Intuitionistic Combinatorial Proofs. In LICS, 2019.

17. Dominic J. D. Hughes. Proofs without syntax. Annals of Mathematics, 164(3):1065-1076, November 2006.

18. Dominic J. D. Hughes. Towards Hilbert's 24th Problem: Combinatorial Proof Invariants:(Preliminary version). Electronic Notes in Theoretical Computer Science, 165:37-63, 2006.

19. Dominic J. D. Hughes. First-order proofs without syntax. arXiv:1906.11236 [math], June 2019.

20. Christian Retoré. Pomset logic: A non-commutative extension of classical linear logic. In Typed Lambda Calculi and Applications, pages 300-318. Springer, 1997.

21. Christian Retoré. Handsome Proof-Nets: REB-Graphs, Perfect Matchings and Series-Parallel Graphs. PhD thesis, INRIA, 1999.

22. Christian Retoré. Handsome proof-nets: Perfect matchings and cographs. Theoretical Computer Science, 294(3):473-488, 2003.

23. Lutz Straßburger. Linear Logic and Noncommutativity in the Calculus of Structures. PhD thesis, Technischen Universität Dresden, 2003. 
24. Lutz Straßburger. MELL in the calculus of structures. Theoretical Computer Science, 309(1):213-285, 2003.

25. Lutz Straßburger. A characterization of medial as rewriting rule. In International Conference on Rewriting Techniques and Applications, pages 344-358. Springer, 2007.

26. Lutz Straßburger. Combinatorial flows and their normalisation. In LIPIcs-Leibniz International Proceedings in Informatics, volume 84. Schloss Dagstuhl-LeibnizZentrum fuer Informatik, 2017.

27. Lutz Straßburger. The problem of proof identity, and why computer scientists should care about Hilbert's 24th problem. Philosophical Transactions of the Royal Society A, 377(2140):20180038, 2019.

28. Lutz Straßburger and Alessio Guglielmi. A System of Interaction and Structure IV: The Exponentials and Decomposition. ACM Transactions on Computational Logic, 12(4):1-39, July 2011.

29. Rüdger Thiele. Hilbert's twenty-fourth problem. American Mathematical Monthly, 110(1):1-24, January 2003. 